\title{
Exploitation of the genetic variability in the genotype of Egyptian Wheat by studding Molecular Markers, Molecular Docking and Nano Composites. Regulation of leaf and stem rust.
}

${ }^{*}$ Hanaa S. Omar, B Mohamed S. Sedeek, C Israa. M. Shamkh E Nour El-Houda A. Reyad

This manuscript has been withdrawn by the authors as it was submitted and made public without the full consent of all the authors. Therefore, the authors do not wish this work to be cited as reference for the project. If you have any questions, please contact the corresponding author. 